\title{
Using an Ordinal Approach to Compare Outcomes Between Vancomycin Versus Ceftaroline or Daptomycin in MRSA Bloodstream Infection
}

\author{
Ashley Barlow · Emily L. Heil · Kimberly C. Claeys (D)
}

Received: December 7, 2020 / Accepted: January 9, 2021 / Published online: January 23, 2021

(c) The Author(s) 2021

\begin{abstract}
Introduction: Vancomycin remains first-line therapy for methicillin-resistant Staphylococcus aureus (MRSA) blood stream infections (BSI); however, its toxicity and reported clinical failures are well established. Binary efficacy endpoints evaluating alternative anti-MRSA therapies leave clinicians deciphering between segregated clinical and safety outcomes and do not provide a comprehensive patient-centered picture of comparative therapies. This study aimed to apply a novel methodology, desirability of outcomes ranking (DOOR), to compare anti-MRSA therapies.

Methods: This was a single-centered, retrospective, cohort of adult patients with MRSA BSI that received vancomycin, daptomycin, or ceftaroline. A previously developed DOOR for $S$. aureus BSI was adjusted and applied to this cohort to compare vancomycin-treated versus daptomycin/ceftaroline-treated patients. The DOOR had five mutually exclusive ranks: (1) alive without treatment failure, infectious complications, or grade 4 adverse events (AEs);
\end{abstract}

A. Barlow · E. L. Heil · K. C. Claeys $(\bowtie)$

University of Maryland Medical Center, Baltimore, MD, USA

e-mail: kclaeys@rx.umaryland.edu

E. L. Heil · K. C. Claeys

University of Maryland School of Pharmacy,

Baltimore, MD, USA
(2) alive with any one of treatment failure, infectious complications, or grade $4 \mathrm{AE}$; (3) alive with two of treatment failure, infectious complications, or grade $4 \mathrm{AE}$; (4) alive with all three treatment failure, infectious complications, or grade $4 \mathrm{AE}$; or (5) deceased.

Results: A total of 43 vancomycin-treated and 13 daptomycin/ceftaroline-treated patients were included. Baseline clinical characteristics were similar, except for higher median serum creatinine in the daptomycin/ceftaroline cohort (0.76 [IQR 0.57, 1.11] vs 1.36 [IQR 1.09, 1.91] $\mathrm{mg} / \mathrm{dL}, P=0.03)$. Patients in the daptomycin/ ceftaroline cohort had a $92 \%$ probability of better outcome using DOOR methodology. Patients treated with daptomycin/ceftaroline experienced less MRSA BSI persistence $(0 \%$ vs $13.9 \%)$, MRSA BSI recurrence (7.8\% vs $25.6 \%)$, grade 4 AEs (23.1\% vs $46.5 \%)$, and in-hospital mortality (0\% vs $9.3 \%)$.

Conclusions: Although limited by sample size, this study demonstrates the potential of DOOR to produce valuable, patient-centered results. Clinicians are encouraged to become familiar with appropriate use and interpretation of DOOR methodology as it will become an increasingly common endpoint in clinical trials.

Keywords: Daptomycin; Desirability of outcomes ranking; S.aureus bacteremia; Vancomycin 


\section{Key Summary Points}

Vancomycin remains the mainstay therapy for methicillin-resistant $S$. aureus (MRSA) bloodstream infection (BSI), although daptomycin and ceftaroline may improve patient outcomes.

Study endpoints separate clinical outcomes and safety metrics and fail to provide comprehensive patient-centered comparisons of alternative therapies.

The desirability of outcomes ranking (DOOR) methodology ordinally ranks combinations of clinical and safety outcomes in mutually exclusive categories to determine overall probability of better outcome with one therapy versus another.

Infectious diseases experts have developed and validated a consensus-based DOOR for $S$. aureus BSI. This current study adjusted this DOOR for specific anti-MRSA therapies and applied it to a retrospective cohort of patients with MRSA BSI.

In this proof-in-concept cohort study, treatment of MRSA BSI with daptomycin or ceftaroline was associated with a 92\% probability of overall better patient outcomes compared to treatment with vancomycin.

\section{DIGITAL FEATURES}

This article is published with digital features, including a summary slide, to facilitate understanding of the article. To view digital features for this article go to https://doi.org/10.6084/ m9.figshare.13547663.

\section{INTRODUCTION}

Bloodstream infections (BSI) caused by methicillin resistant Staphylococcus aureus (MRSA) are

associated with substantial morbidity and mortality [1]. Although vancomycin (VAN) remains the standard therapy for MRSA BSI, nephrotoxicity and risk of clinical failure with minimum inhibitory concentrations (MIC) greater than $1 \mathrm{mg} / \mathrm{L}$ represent practical limitations [1-5]. Alternative MRSA antibiotics, in particular daptomycin (DAP) and ceftaroline (CPT), can help overcome these shortcomings. Literature comparing VAN to these agents for MRSA BSI have demonstrated superior outcomes with DAP compared to VAN and comparable efficacy with CPT compared to VAN $[2,5,6]$. The use of binary efficacy endpoints, such as clinical cure versus failure, does not incorporate the balance of competing benefits and risks to the individual patient, however.

A novel statistical methodology, the desirability of outcomes ranking (DOOR), has been previously applied in infectious diseases literature to provide clinicians with an improved assessment of risks versus benefits of alternative antibiotic therapies [7]. The DOOR methodology uses rankings to construct patient-centered assessments of clinical outcomes and adverse events between two interventions or therapies to better inform clinical decision-making [7-9]. In brief, each ordinal ranking contains mutually exclusive categories of patient outcomes, combining both efficacy and safety metrics in each rank. The worst rank is often reserved for inpatient mortality, while the best rank is often a combination of clinical cure of infection without development of antibiotic-associated adverse drug events. These rankings can be used for a visual comparison of patient-centered outcomes or, through a series of pairwise comparisons, one can assess the probability of an overall better outcome with one therapy versus another. The primary goal of this proof-inconcept study was to apply a previously validated DOOR for S. aureus BSI (SAB) to a retrospective dataset of patents with MRSA BSI and compare outcomes between those patients that received VAN versus DAP or CPT. 


\section{METHODS}

\section{Study Design, Setting, and Population}

A single-center retrospective cohort study conducted at University of Maryland Medical Center (UMMC) for patients at least 18 years old with confirmed MRSA BSI between November 15, 2015 and August 1, 2019. UMMC is a 757-bed tertiary academic medical center with specialties including shock trauma, solid organ transplant, and oncology. Inclusion criteria were patients with MRSA BSI, confirmed by at least one positive blood culture, treated with VAN, DAP, or CPT for $72 \mathrm{~h}$ or more. Exclusion criteria included those with bacterial pneumonia or central nervous system infection, death within $72 \mathrm{~h}$ of treatment initiation, polymicrobial infection, initial receipt of renal replacement therapy, alternative MRSA antibiotics for more than $72 \mathrm{~h}$, or combination MRSA antibiotics for $72 \mathrm{~h}$ or more. All MRSA BSI requires an infectious diseases (ID) consult and both DAP and CPT were restricted to ID use only; therefore, all patients in this study were being followed by ID consults. This study was approved by the University of Maryland institutional review board with a waiver of informed consent.

\section{Study Definitions}

The SAB DOOR is a previously validated expertguided DOOR applied to S. aureus BSI [8]. The main components of the SAB DOOR include clinical resolution, lack of infectious complications, and lack of antibiotic-related adverse events (AE). With respect to clinical resolution of MRSA BSI, patients could not have any of the following: relapse or readmission attributable to MRSA BSI or persistent MRSA BSI of greater than 5 days. Infectious complications included development of resistance to one or more study drugs or newly identified metastatic focus of infection. The $\mathrm{AE}$ criteria were modified to reflect grade $4 \mathrm{AE}$ from VAN, DAP, or CPT. Grade $4 \mathrm{AE}$ for VAN was defined as Kidney Disease Improving Global Outcomes stage 2 or 3 acute kidney injury (AKI), platelets $75 \times 10^{9} /$ $\mu \mathrm{L}$ or less, or absolute neutrophil count (ANC) less than 1500 cells/ $\mu \mathrm{L}$ [10. For DAP descriptors of rhabdomyolysis (creatinine phosphokinase greater than 2000 units/L or five times the upper limit of normal with muscle symptoms) were assessed [11]. For CPT, severe hypersensitivity reactions, platelets $75 \times 10^{9} \mu \mathrm{L}$ or less, or ANC less than 1500 cells/ $\mu \mathrm{L}$ was assessed [12]. The five possible ranks were (1) alive without treatment failure, infectious complications, or grade $4 \mathrm{AEs}$; (2) alive with any one of treatment failure, infectious complications, or grade $4 \mathrm{AE}$; (3) alive with any two of treatment failure, infectious complications, or grade $4 \mathrm{AE}$; (4) alive with all three of treatment failure, infectious complications, or grade $4 \mathrm{AE}$; or (5) deceased. Therefore, a higher DOOR ranking was associated with better clinical outcomes (Table 1). All data was collected from electronic medical records based on inpatient documentation only.

\section{Statistical Analysis}

Descriptive statistics included frequencies with proportions, and medians with interquartile ranges (IQRs). Binary comparisons between those that received VAN versus DAP or CPT were completed using chi-squared or Fisher's exact test for nominal variables and Mann Whitney $U$ test for continuous variables. A $P$ value less than 0.05 was considered statistically significant. In order to compute the probability of a better DOOR with one treatment over another, a series of pairwise comparisons

Table 1 Explanation of DOOR endpoints and ordinal ranking scheme

\begin{tabular}{lll}
\hline Rank & Alive & $\begin{array}{l}\text { Number of grade } 4 \text { adverse events, } \\
\text { infectious complications experienced, or } \\
\text { treatment failure }\end{array}$ \\
\hline 1 & Yes & 0 of 3 \\
2 & Yes & 1 of 3 \\
3 & Yes & 2 of 3 \\
4 & Yes & 3 of 3 \\
5 & No & Any \\
\hline
\end{tabular}


Table 2 Baseline characteristics and clinical outcomes

\begin{tabular}{|c|c|c|c|}
\hline & VAN $(n=43)$ & DAP $(n=10)$ CPT $(n=3)$ & $P$ value \\
\hline \multicolumn{4}{|l|}{ Baseline characteristics } \\
\hline Age, median (range) & $53(25,89)$ & $58(29,80)$ & 0.34 \\
\hline Sex, male, $n(\%)$ & $23(53.5)$ & $10(77)$ & 0.2 \\
\hline Body weight, kg, median (IQR) & $68(59,86.3)$ & $88(72,99)$ & 0.06 \\
\hline Serum creatinine, mg/dL, median, (IQR) & $0.76(0.57,1.11)$ & $1.36(1.09,1.91)$ & 0.03 \\
\hline Intensive care unit, $n(\%)$ & $5(11.6)$ & $1(7.7)$ & 0.99 \\
\hline \multicolumn{4}{|l|}{ Source of MRSA BSI, $n(\%)$} \\
\hline Endocarditis & $12(27.9)$ & $4(30.8)$ & 0.72 \\
\hline Skin and soft tissue & $10(23.3)$ & $3(23.1)$ & 0.99 \\
\hline Intra-abdominal & $2(4.7)$ & $1(7.8)$ & 0.53 \\
\hline Bone-joint & $11(25.6)$ & $2(15.4)$ & 1.00 \\
\hline Central line & $4(9.3)$ & $1(7.8)$ & 1.00 \\
\hline Unknown & $4(9.3)$ & $2(15.4)$ & 0.61 \\
\hline \multicolumn{4}{|l|}{ Concomitant antibiotics, $n(\%)$} \\
\hline Piperacillin-tazobactam & $14(32.5)$ & $5(38.4)$ & 1.00 \\
\hline Aminoglycoside & $2(4.7)$ & $1(7.8)$ & 0.55 \\
\hline Cefepime & $10(25.6)$ & $3(23.1)$ & 1.00 \\
\hline Ceftriaxone & $5(11.6)$ & 0 & 0.58 \\
\hline Rifampin & $5(11.6)$ & $1(7.8)$ & 1.00 \\
\hline Other & $5(11.6)$ & $3(23.1)$ & 0.37 \\
\hline Days concomitant antibiotics, median (IQR) & $2(1.5,3.5)$ & $2(1,3)$ & 0.26 \\
\hline \multicolumn{4}{|l|}{ Clinical outcomes } \\
\hline Duration of hospitalization (days), median (IQR) & $14(7,21.5)$ & $18(11,26)$ & 0.4 \\
\hline Inpatient DOT (days), median (IQR) & $14(6.5,19)$ & $12(8,14)$ & 0.51 \\
\hline Total DOT, median (IQR) & $30(15,43)$ & $19(13,40)$ & 0.97 \\
\hline AKI development, $n(\%)$ & $20(46.5)$ & $3(23.1)$ & 0.2 \\
\hline Initiation of RRT, $n$ (\%) & $7(16.3)$ & $0(0)$ & 0.18 \\
\hline CPK elevations, $n(\%)$ & $\mathrm{N} / \mathrm{A}$ & $1(7.8)$ & $\mathrm{N} / \mathrm{A}$ \\
\hline Hematologic, $n(\%)^{\mathrm{a}}$ & $7(16.3)$ & $0(0)$ & 0.18 \\
\hline MRSA BSI persistence, $n(\%)^{\mathrm{b}}$ & $6(13.9)$ & $0(0)$ & 0.31 \\
\hline MRSA BSI recurrence, $n(\%)^{c}$ & $11(25.6)$ & $1(7.8)$ & 0.25 \\
\hline 90-day MRSA readmission, $n$ (\%) & $17(39.5)$ & $3(23.1)$ & 0.99 \\
\hline In-hospital mortality, $n(\%)$ & $4(9.3)$ & $0(0)$ & 0.56 \\
\hline
\end{tabular}


Table 2 continued

\begin{tabular}{llll}
\hline & VAN $(n=43)$ & DAP $(n=10)$ CPT $(n=3)$ & $P$ value \\
\hline 30-day all-cause mortality, $n(\%)$ & $4(9.3)$ & $0(0)$ & 0.56 \\
\hline
\end{tabular}

IQR interquartile range, MRSA methicillin resistant Staphylococcus aureus, BSI blood stream infection, DOT duration of treatment, $A K I$ acute kidney injury, $R R T$ renal replacement therapy, $C P K$ creatinine phosphokinase, $N / A$ not applicable

a Hematologic defined as a composite of thrombocytopenia or neutropenia

b MRSA BSI persistence defined as positive blood cultures for $\geq 5$ days

c MRSA recurrence defined as positive MRSA blood culture $\geq 48 \mathrm{~h}$ after negative blood culture

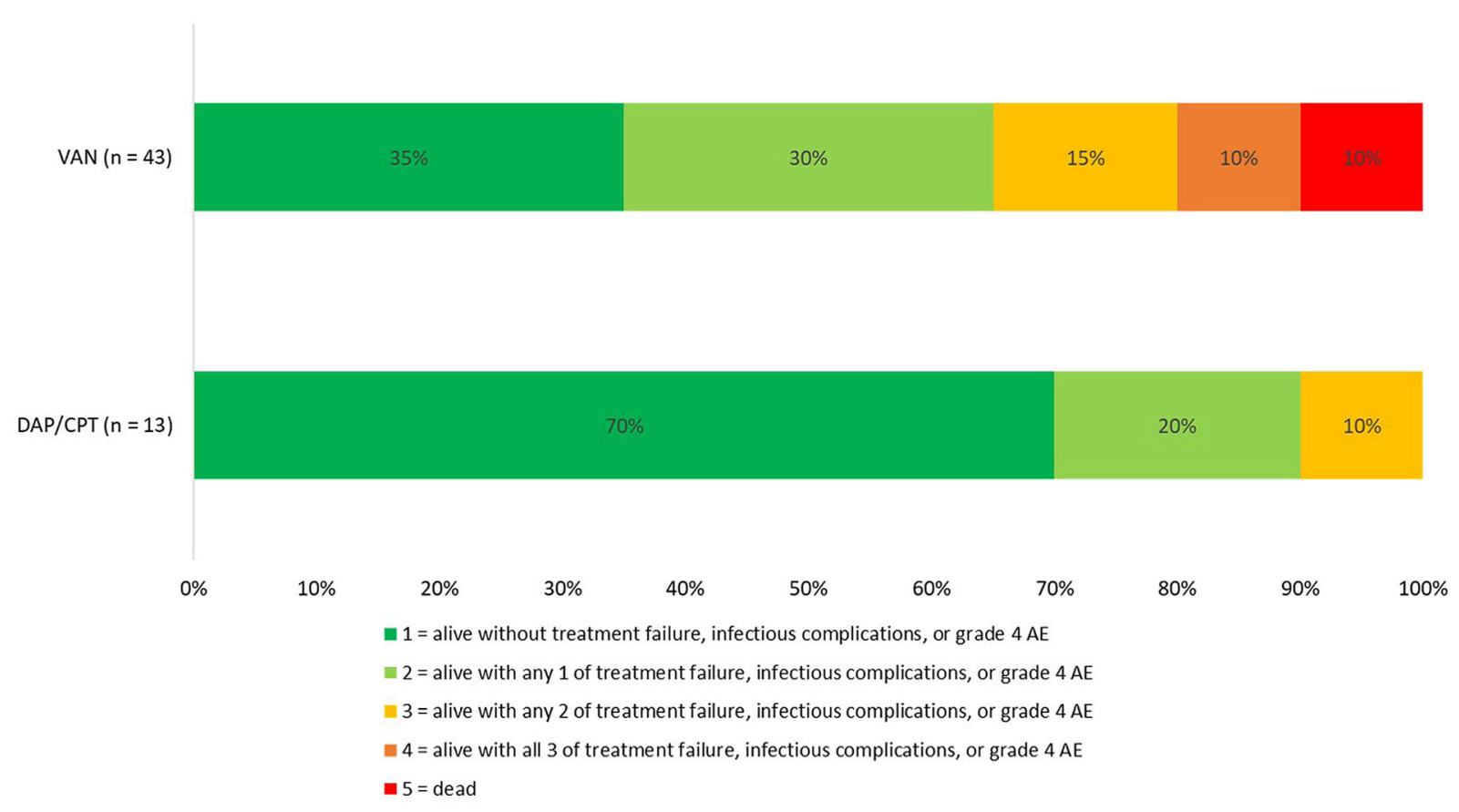

Fig. 1 Comparison of DOOR vancomycin vs ceftaroline/daptomycin

between treatment groups was completed [7]. All analysis was completed using Microsoft Excel.

\section{RESULTS}

A total of 125 patients were screened for study inclusion. Among these, 69 patients were excluded for the following reasons: age less than 18 years $(n=2)$, pneumonia source $(n=9)$, alternative MRSA therapy for more than $72 \mathrm{~h}$ $(n=15)$, received less than $48 \mathrm{~h}$ of inpatient therapy $(n=27)$, polymicrobial infection $(n=9)$, and history of renal dialysis $(n=7)$. The final study population consisted of 43 patients in the VAN group compared to 13 patients in the DAP/CPT group (10 DAP and $3 \mathrm{CPT}$ ). Among the DAP/CPT patients the majority $(n=10)$ initially received VAN therapy for a median of 2 (IQR 1, 2.25) days. All patients in this study had complicated MRSA BSI. Differences in baseline characteristics are documented in Table 2. Patients in the DAP/CPT cohort had higher median baseline serum creatinine compared to the VAN cohort. The VAN 
MIC was $\leq 1 \mathrm{mg} / \mathrm{dL}$ for all patients in the VAN cohort versus $92.3 \%$ in the DAP/CPT cohort. The median VAN trough and AUC/MIC at $48 \mathrm{~h}$ were 15.4 (IQR $13.4,18.3$ ) $\mathrm{mg} / \mathrm{dL}$ and 516 (IQR $452,554)$, respectively. Median DAP doses were 6 (IQR 6,8$) \mathrm{mg} / \mathrm{kg}$ and CPT was dosed at $600 \mathrm{mg}$ every $8 \mathrm{~h}(n=2)$ or every $12 \mathrm{~h}(n=1)$. Upon discharge many VAN patients were continued on outpatient therapy $(n=29,67 \%)$, primarily on VAN $(n=15)$ or DAP $(n=11)$. Among DAP/ CPT patients, eight $(61.5 \%)$ were discharged on outpatient antibiotics, with DAP being the most common agent $(n=7)$. Those in the DAP/CPT cohort had a lower DOOR compared to VAN (Fig. 1); DAP/CPT patients had a $92 \%$ probability of better outcome compared to those that received VAN. This difference in overall outcomes was driven by higher rates of MRSA BSI persistence (13.9\% vs $0 \%$ ) and MRSA BSI recurrence $(25.6 \%$ vs $7.8 \%)$ observed with VAN compared to DAP/CPT. Overall adverse events were higher in the VAN cohort compared to the DAP/CPT cohort; primarily nephrotoxicity (46.5\% versus $23.1 \%$ ) and requirement for receipt of renal replacement therapy (16.3\% versus 0\%). Lastly, in-hospital mortality was higher in the VAN cohort compared to the DAP/ CPT cohort (9.3\% versus $0 \%)$.

\section{DISCUSSION}

With the application of a modified SAB DOOR, we observed improved clinical outcomes without the competing risk of increased toxicity among the DAP/CPT cohort compared to the VAN cohort. Results from Murray et al. of DAP versus VAN in MRSA BSI with elevated VAN MIC align with our findings, showing lower 30 -day mortality $(3.5 \%$ vs $12.9 \% ; P=0.047)$ and less persistent bacteremia $(18.8 \%$ versus $42.4 \% P=0.001)$ in those treated with DAP [5]. Claeys et al. demonstrated higher rates of clinical failure $(45.0 \%$ versus $29.0 \% ; P=0.007)$ and all-cause 30 -day mortality (15.3\% versus $6.1 \%$; $P=0.024)$ with VAN compared to DAP, regardless of MIC [2]. Despite a higher baseline creatinine clearance in the current DAP/CPT cohort, higher rates of nephrotoxicity and need for renal replacement therapy were observed in the
VAN cohort. This is consistent with previous data for gram-positive infections where VAN was associated with higher rates nephrotoxicity compared to DAP $(23.3 \%$ vs $6.3 \%, P=0.006)$ and receipt of VAN was an independent risk factor for nephrotoxicity [13].

A major strength of this study was the incorporation of a validated DOOR that was generated by expert consensus [8]. With the recognition that providers place significant value on patient-centered outcomes beyond simple binaries of clinical cure, the DOOR methodology helps fulfill this unmet need. The current study is hypothesis-generating given the limitations of being single-centered and retrospective in nature. Additionally, a major limitation is the small sample size and inability to calculate an a priori sample size requirement. Although we used a previously validated DOOR, the application of the ranking in a retrospective fashion limits the strength of the results as confounding by indication is still possible. The baseline imbalance in initial serum creatinine between groups and insufficient description of other nephrotoxic therapies group may have contributed to differences in nephrotoxicity. Despite these limitations, nephrotoxicity has been a reproducible and established concern with VAN. Within our institution, VAN is first line for MRSA BSI with transition to DAP or CPT only on suspicion of clinical failure or development of nephrotoxicity. This led to difficultly in identifying a large DAP/CPT cohort that was not treated with an extended duration of VAN prior. Among the DAP/CPT cohort, the majority were initiated on VAN. Although VAN was given for less than $72 \mathrm{~h}$, this initial receipt could additionally lead to confounding of the outcome. Additionally, our institution transitioned to VAN AUC/MIC monitoring in 2017 which may have influenced the rates of VAN nephrotoxicity. Finally, the three patients with persistent MRSA BSI had endocarditis which could reflect a failure of source control rather than antibiotic failure. Results of this study should be interpreted with caution, given the limited sample size; however, they provide proof-inconcept regarding the value of DOOR methodology. 


\section{CONCLUSION}

Application of DOOR methodology has the potential to produce valuable, patient-centered outcomes. The SAB DOOR should be applied in future trials to better assess the risk/benefit trade-off of treatment of MRSA BSI from a patient perspective. Clinicians are encouraged to become familiar with appropriate use and interpretation of DOOR methodology as it will likely become an increasingly common endpoint among infectious diseases research and clinical trials.

\section{ACKNOWLEDGEMENTS}

Funding. No funding or sponsorship was received for this study or publication of this article.

Authorship. All named authors meet the International Committee of Medical Journal Editors (ICMJE) criteria for authorship for this article and take responsibility for the integrity of the published work.

Disclosures. Ashley Barlow, Emily L. Heil and Kimberly C. Claeys have nothing to disclose.

Compliance with Ethics Guidelines. This study was approved by the University of Maryland institutional review board with a waiver of informed consent.

Data Availability. The datasets generated during and/or analyzed during the current study are available from the corresponding author on reasonable request.

Open Access. This article is licensed under a Creative Commons Attribution-NonCommercial 4.0 International License, which permits any non-commercial use, sharing, adaptation, distribution and reproduction in any medium or format, as long as you give appropriate credit to the original author(s) and the source, provide a link to the Creative Commons licence, and indicate if changes were made. The images or other third party material in this article are included in the article's Creative Commons licence, unless indicated otherwise in a credit line to the material. If material is not included in the article's Creative Commons licence and your intended use is not permitted by statutory regulation or exceeds the permitted use, you will need to obtain permission directly from the copyright holder. To view a copy of this licence, visit http://creativecommons.org/licenses/by$\mathrm{nc} / 4.0 /$.

\section{REFERENCES}

1. Liu C, Bayer A, Cosgrove SE, et al. Clinical practice guidelines by the Infectious Diseases Society of America for the treatment of methicillin-resistant Staphylococcus aureus infections in adults and children. Clin Infect Dis. 2011;52(3):e18-55. https:// doi.org/10.1093/cid/ciq146.

2. Claeys KC, Zasowski EJ, Casapao AM, et al. Daptomycin improves outcomes regardless of vancomycin MIC in a propensity-matched analysis of methicillin-resistant Staphylococcus aureus bloodstream infections. Antimicrob Agents Chemother. 2016;60(10):5841-8. https://doi.org/10.1128/AAC. 00227-16.

3. Moise PA, Culshaw DL, Wong-Beringer A, et al. Comparative effectiveness of vancomycin versus daptomycin for MRSA bacteremia with vancomycin MIC $>1 \mathrm{mg} / \mathrm{L}$ : a multicenter evaluation. Clin Ther. 2016;38(1):16-30. https://doi.org/10.1016/j. clinthera.2015.09.017.

4. Moore CL, Osaki-Kiyan P, Haque NZ, Perri MB, Donabedian S, Zervos MJ. Daptomycin versus vancomycin for bloodstream infections due to methicillin-resistant Staphylococcus aureus with a high vancomycin minimum inhibitory concentration: a case-control study. Clin Infect Dis. 2012;54(1):51-8. https://doi.org/10.1093/cid/cir764.

5. Murray KP, Zhao JJ, Davis SL, et al. Early use of daptomycin versus vancomycin for methicillin-resistant Staphylococcus aureus bacteremia with vancomycin minimum inhibitory concentration $>1$ mg/L: a matched cohort study. Clin Infect Dis. 2013;56(11):1562-9. https://doi.org/10.1093/cid/ cit112.

6. Arshad S, Huang V, Hartman P, Perri MB, Moreno D, Zervos MJ. Ceftaroline fosamil monotherapy for methicillin-resistant Staphylococcus aureus 
bacteremia: a comparative clinical outcomes study. Int J Infect Dis. 2017;57:27-31. https://doi.org/10. 1016/j.ijid.2017.01.019.

7. Evans SR, Rubin D, Follmann D, et al. Desirability of outcome ranking (DOOR) and response adjusted for duration of antibiotic risk (RADAR). Clin Infect Dis. 2015;61(5):800-6. https://doi.org/10.1093/cid/ civ495.

8. Doernberg SB, Tran TTT, Tong SYC, et al. Good studies evaluate the disease while great studies evaluate the patient: development and application of a desirability of outcome ranking endpoint for Staphylococcus aureus bloodstream infection. Clin Infect Dis. 2019;68(10):1691-8. https://doi.org/10. 1093/cid/ciy766.

9. Evans SR, Follmann D. Using outcomes to analyze patients rather than patients to analyze outcomes: a step toward pragmatism in benefit:risk evaluation. Stat Biopharm Res. 2016;8(4):386-93. https://doi. org/10.1080/19466315.2016.1207561.
10. ANI Pharmaceuticals. Vancomycin hydrochloride product description.. https://www.accessdata.fda. gov/drugsatfda_docs/label/2017/060180s047lbl. pdf. Accessed 27 May 2020.

11. Merck. Highlights of prescribing information: CUBICIN ${ }^{\circledR}$ (daptomycin for injection). https:// www.merck.com/product/usa/pi_circulars/c/ cubicin/cubicin_pi.pdf. Accessed 27 May 2020.

12. Forest Laboratories. Highlights of prescribing information: Teflaro $^{\mathrm{TM}}$ (ceftaroline fosamil). https://www.accessdata.fda.gov/drugsatfda_docs/ label/2011/200327s001lbl.pdf. Accessed 27 May 2020 .

13. Barberán J, Mensa J, Artero A, et al. Factors associated with development of nephrotoxicity in patients treated with vancomycin versus daptomycin for severe gram-positive infections: a practice-based study. Rev Esp Quimioter. 2019;32(1): 22-30. 